\title{
EFFECTS OF FLUID COMPOSITION ON SPHERICAL FLOWS AROUND BLACK HOLES
}

\author{
IndRanil Chattopadhyay ${ }^{1}$ and Dongsu Ryu ${ }^{2,3}$ \\ ${ }^{1}$ ARIES, Manora Peak, Nainital-263129, Uttaranchal, India; indra@aries.ernet.in \\ ${ }^{2}$ Department of Astronomy and Space Science, Chungnam National University, Daejeon 305-764, South Korea; ryu@canopus.cnu.ac.kr \\ Received 2008 May 11; accepted 2008 December 18; published 2009 March 17
}

\begin{abstract}
Steady, spherically symmetric, adiabatic accretion and wind flows around nonrotating black holes were studied for fully ionized, multicomponent fluids, which are described by a relativistic equation of state. We showed that the polytropic index depends on the temperature as well as on the composition of fluids, so the composition is important to the solutions of the flows. We demonstrated that fluids with different composition can produce dramatically different solutions, even if they have the same sonic point, or they start with the same specific energy or the same temperature. Then, we pointed that the Coulomb relaxation times can be longer than the dynamical time in the problem considered here, and discussed the implication.
\end{abstract}

Key words: accretion, accretion disks - black hole physics - hydrodynamics - relativity

\section{INTRODUCTION}

It is generally inferred from observations that the matter falling onto black holes is of very high temperature, both in microquasars (Corbel et al. 2003) as well as in active galactic nuclei (AGNs; Rózańska \& Czerny 2000). The electron temperature around $10^{9} \mathrm{~K}$ and/or the proton temperature around $10^{12} \mathrm{~K}$ or more are accepted as typical values within few tens of the Schwarzschild radius, $r_{s}$, of the central black holes. Moreover, the general theory of relativity demands that the matter crosses the black hole horizon with the speed of light (c). In other words, close to black holes, the matter is relativistic in terms of its bulk speed and/or its temperature. On the other hand, at large distances away from black holes, the matter should be nonrelativistic.

It is also inferred from observations that the astrophysical jets around black hole candidates have relativistic speeds (Biretta et al. 2003). Since the jets originate from the accreting matter very close to black holes, their base could be very hot. At a few hundred Schwarzschild radii above the disk plane, they can expand to very low temperatures but very high speeds (Lorentz factor $\gamma \gtrsim$ a few). And as the fast moving matter of the jets hits the ambient medium and drastically slows down to form shocks and hot spots, once again the thermal energy increases to relativistic values though the bulk velocity becomes small. Relativistic flows are inferred for gamma-ray bursts (GRBs) too. In the so-called collapsar model scenario (Woosley 1993), the collimated bipolar outflows emerge from deep inside collapsars and propagate into the interstellar medium (ISM), producing GRBs and afterglows. In such model, these collimated outflows are supposed to achieve Lorentz factors $\gamma \gtrsim$ 100.

It is clear in the above examples that as a fluid flows onto a black hole or away from it, there are one or more transitions from the nonrelativistic regime to the relativistic one or vice versa. It has been shown by quite a few authors that to describe such trans-relativistic fluid, the equation of state $(\mathrm{EoS})$ with a fixed adiabatic index $\Gamma$ ( $=c_{p} / c_{v}$, the ratio of specific heats) is inadequate and the relativistically correct EoS (Chandrasekhar

\footnotetext{
3 Corresponding author.
}

1938; Synge 1957) should be used (e.g., Taub 1948; Mignone et al. 2005; Ryu et al. 2006).

A fluid is said to be thermally relativistic, if its thermal energy is comparable to or greater than its rest-mass energy, i.e., if $k T \gtrsim m c^{2}$. The thermally nonrelativistic regime is $k T \ll m c^{2}$. Here, $T$ is the temperature, $k$ is the Boltzmann constant, and $m$ is the mass of the particles that constitute the fluid. So it is not just the temperature that determines a fluid to be thermally relativistic, but it is the ratio, $T / m$, that determines it. Therefore, together with the temperature, the composition of the fluid (i.e., either the fluid is composed of electron-positron pairs, or electrons and protons, or some other combinations) will determine whether the fluid is in the thermally relativistic regime or not.

The study of relativistic flows around compact objects including black holes was started by Michel (1972). It was basically recasting the transonic accretion and wind solutions around Newtonian objects obtained by Bondi (1952) into the framework of the general theory of relativity. Since then, a number of authors have addressed the problem of relativistic flows around black holes, each focusing on its various aspects (e.g., Blumenthal \& Mathews 1976; Ferrari \& Trussoni 1985; Chakrabarti 1996a; Das 2001, 2002; Meliani et al. 2004; Barai et al. 2006; Fukumura \& Kazanas 2007; Mandal et al. 2007). Barring a few exceptions (e.g., Blumenthal \& Mathews 1976; Meliani et al. 2004), most of these studies used the EoS with a fixed $\Gamma$, which, as we have noted, is incapable of describing a fluid from infinity to the horizon. Blumenthal \& Mathews (1976) for the first time calculated the spherical accretion and wind solutions around Schwarzschild black holes, while using an approximate EoS for the single-component relativistic fluid (Mathews 1971). Meliani et al. (2004) modified the EoS used by Blumenthal \& Mathews (1976) to obtain thermally driven spherical winds with relativistic terminal speeds. However, there has been no extensive study of the effects of fluid composition on the solutions of transonic flows around black holes. We in this paper investigate the effects.

The paper is organized as follows. In the next section, we present the governing equations including the EoS. In Section 3 , we present the sonic-point properties. In Section 4, we present the accretion and wind solutions. In Section 5, we discuss the validity of our relativistic EoS. Discussion and concluding remarks are presented in the last section. 


\section{ASSUMPTIONS AND EQUATIONS}

To ensure that the effects of fluid composition are clearly presented, we keep our model of accretion and wind as simple as possible. We consider adiabatic, spherical flows onto Schwarzschild black holes. The space time is described by the Schwarzschild metric

$$
\begin{aligned}
d s^{2}= & -\left(1-\frac{2 G M_{B}}{c^{2} r}\right) c^{2} d t^{2}+\left(1-\frac{2 G M_{B}}{c^{2} r}\right)^{-1} d r^{2} \\
& +r^{2} d \theta^{2}+r^{2} \sin ^{2} \theta d \phi^{2}
\end{aligned}
$$

where $r, \theta, \phi$ are the usual spherical coordinates, $t$ is the time, and $M_{B}$ is the mass of the central black hole. Although AGNs and micro-quasars are in general powered by rotating flows, studies of spherical flows are not entirely of pedagogic interest. For instance, such studies can throw light on the nature of accretions onto isolated black holes in low angular momentum and cold clouds. In addition, hot spherical flows may mimic accretions very close to black holes, where the accreting matter is expected to be of low angular momentum, hot, and with strong advection. Nonconservative processes and magnetic fields are ignored, too. by

The energy-momentum tensor of a relativistic fluid is given

$$
T^{\mu \nu}=(e+p) u^{\mu} u^{v}+p g^{\mu \nu},
$$

where $e$ and $p$ are the energy density and gas pressure, respectively, all measured in the local frame. The four velocities are represented by $u^{\mu}$. The equations governing fluid dynamics are given by

$$
T_{; v}^{\mu v}=0 \text { and }\left(n u^{v}\right)_{; \nu}=0,
$$

where $n$ is the particle number density of the fluid measured in the local frame.

\subsection{EoS For Single-Component Fluids}

Equation (3) is essentially five independent equations, while the number of variables are six. This anomaly in fluid dynamics is resolved by a closure relation between $e, p$, and $n$ (or the mass density $\rho=n m$ ), and this relation is known as the EoS. The EoS for single-component relativistic fluids, which are in thermal equilibrium, has been known for a while, and is given by

$$
\frac{e+p}{\rho c^{2}}=\frac{K_{3}\left(\rho c^{2} / p\right)}{K_{2}\left(\rho c^{2} / p\right)}
$$

(Chandrasekhar 1938; Synge 1957). Here, $K_{2}$ and $K_{3}$ are the modified Bessel functions of the second kind of order 2 and 3, respectively.

Owing to simplicity, however, the most commonly used EoS has been the one with a fixed $\Gamma$, which is written as

$$
e=\rho c^{2}+\frac{p}{\Gamma-1} .
$$

As noted in Section 1, this EoS, which admits the superluminal sound speed, is not applicable to all ranges of temperature (Mignone et al. 2005; Ryu et al. 2006). Here, we adopt an approximate EoS

$$
e=\rho c^{2}+p\left(\frac{9 p+3 \rho c^{2}}{3 p+2 \rho c^{2}}\right)
$$

which reproduces very closely the relativistically correct EoS in Equation (4a), better than the one proposed by Mathews (1971)

$$
p=\frac{\rho c^{2}}{3}\left(\frac{e}{\rho c^{2}}-\frac{\rho c^{2}}{e}\right) .
$$

A comparative study of various EoS's for single-component relativistic fluids was presented in Ryu et al. (2006).

\subsection{EoS For Multicomponent Fluids}

We consider fluids which are composed of electrons, positrons, and protons. Then the number density is given by

$$
n=\Sigma n_{i}=n_{e^{-}}+n_{e^{+}}+n_{p^{+}},
$$

where $n_{e^{-}}, n_{e^{+}}$, and $n_{p^{+}}$are the electron, positron, and proton number densities, respectively. Charge neutrality demands that

$$
n_{e^{-}}=n_{e^{+}}+n_{p^{+}} \quad \Rightarrow \quad n=2 n_{e^{-}} \quad \text { and } \quad n_{e^{+}}=n_{e^{-}}(1-\xi) \text {, }
$$

where $\xi=n_{p^{+}} / n_{e^{-}}$is the relative proportion of protons. The mass density is given by

$$
\rho=\Sigma n_{i} m_{i}=n_{e^{-}} m_{e}\left\{2-\xi\left(1-\frac{1}{\eta}\right)\right\},
$$

where $\eta=m_{e} / m_{p}$, and $m_{e}$ and $m_{p}$ are the electron and proton masses, respectively. For single-temperature fluids, the isotropic pressure is given by

$$
p=\Sigma p_{i}=2 n_{e^{-}} k T .
$$

As our EoS for multicomponent fluids, we adopt

$$
e=\Sigma e_{i}=\Sigma\left[n_{i} m_{i} c^{2}+p_{i}\left(\frac{9 p_{i}+3 n_{i} m_{i} c^{2}}{3 p_{i}+2 n_{i} m_{i} c^{2}}\right)\right] .
$$

The nondimensional temperature is defined with respect to the electron rest-mass energy, $\Theta=k T /\left(m_{e} c^{2}\right)$. With Equations (5a) $-(5 d)$, the expression of the energy density in Equation (5e) simplifies to

$$
e=n_{e^{-}} m_{e} c^{2} f
$$

where

$$
f=(2-\xi)\left[1+\Theta\left(\frac{9 \Theta+3}{3 \Theta+2}\right)\right]+\xi\left[\frac{1}{\eta}+\Theta\left(\frac{9 \Theta+3 / \eta}{3 \Theta+2 / \eta}\right)\right] .
$$

The expression of the polytropic index for single-temperature fluids is given by

$$
N=\frac{T}{p} \Sigma n_{i} \frac{d \Phi_{i}}{d T}=\frac{1}{2} \frac{d f}{d \Theta},
$$

where

$$
\Phi_{i}=\frac{e_{i}}{n_{i}}=m_{i} c^{2}+k T \frac{9 k T+3 m_{i} c^{2}}{3 k T+2 m_{i} c^{2}}
$$

is the energy density per particle of each component. The effective adiabatic index is calculated by

$$
\Gamma=1+\frac{1}{N}
$$



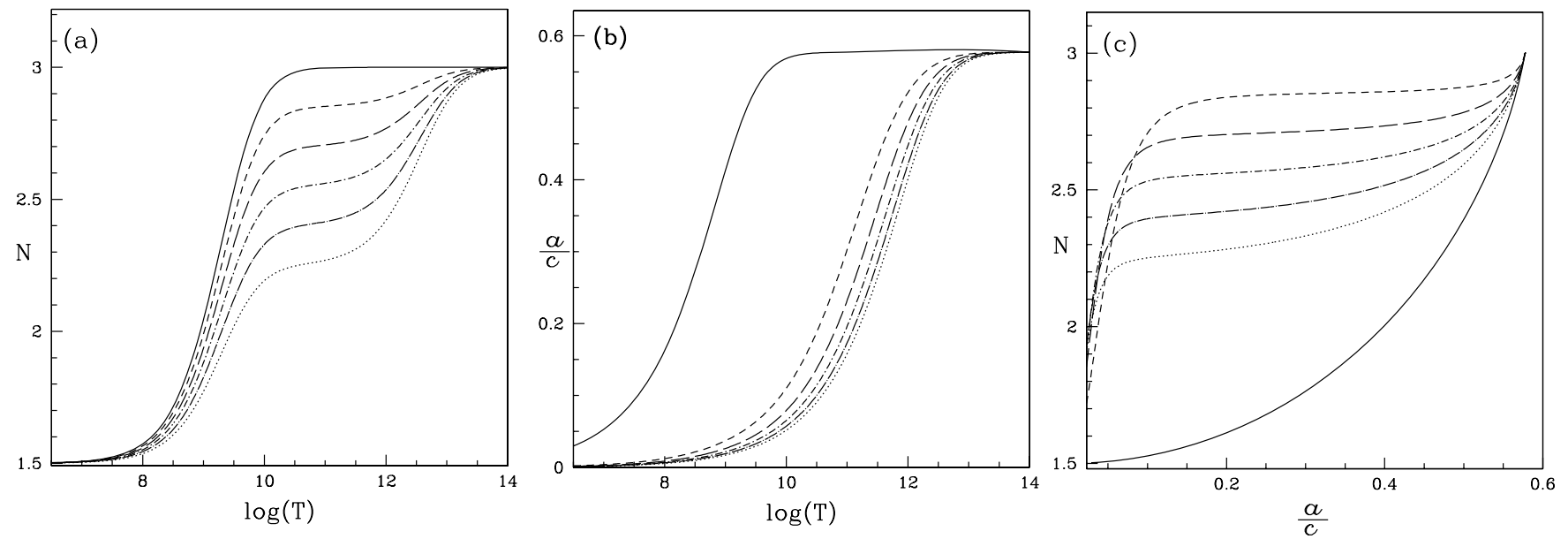

Figure 1. (a) Polytropic index as a function of the temperature, $(b)$ sound speed as a function of the temperature, and $(c)$ polytropic index as a function of the sound speed, for multicomponent relativistic fluids of different composition with proton proportions of $\xi=0$ (solid), 0.2 (dashed), 0.4 (long dashed), 0.6 (dashed-dotted), 0.8 (long dashed-dotted), and 1 (dotted). Hereafter, the temperature in figures is given in units of Kelvin.

The definition of the sound speed, $a$, is

$$
\frac{a^{2}}{c^{2}}=\frac{\Gamma p}{e+p}=\frac{2 \Gamma \Theta}{f+2 \Theta} .
$$

The polytropic index $N$ (and also the adiabatic index $\Gamma$ ) is an indicator of the thermal state of a fluid. If $N \rightarrow 3 / 2$ (or $\Gamma \rightarrow 5 / 3$ ), the fluid is called thermally nonrelativistic. On the other hand, if $N \rightarrow 3$ (or $\Gamma \rightarrow 4 / 3$ ), it is called thermally relativistic. For single-component fluids, $N$ and $\Gamma$ are given as a function of the temperature alone (Ryu et al. 2006). For multicomponent fluids, however, not just the temperature, the mass of the constituent particles also determines the thermal state. Hence, the proton proportion, $\xi$, enters as a parameter too.

In Figure 1, we show various thermodynamic quantities and their inter-relations for fluids with different $\xi$. In Figure 1(a) which plots $N$ as a function of $T$, the leftmost (solid) curve represents the electron-positron pair fluid $(\xi=0)$ (hereafter, the $e^{-}-e^{+}$fluid) and the rightmost (dotted) curve represents the electron-proton fluid $(\xi=1)$ (hereafter, the $e^{-}-p^{+}$fluid). In the $e^{-}-e^{+}$fluid, $N \rightarrow 3$ for $k T>m_{e} c^{2}$, while in the $e^{-}-p^{+}$fluid, $N \rightarrow 3$ for $k T>m_{p} c^{2}$. In the intermediate temperature range, $m_{e} c^{2}<k T<m_{p} c^{2}, N$ decreases (i.e., the fluid becomes less relativistic) with the increase of $\xi$. It is because if $\xi$ increases (i.e., the proton proportion increases), the thermal energy required to be in the relativistic regime also increases. By the same reason, at the same $T$, the local sound speed, $a$, decreases as $\xi$ increases, as shown in Figure 1(b). However, in Figure 1(c), it is shown that the relation between $N$ and $a$ is not as simple as the relation between $N$ and $T$. At the same $a, N$ is smallest for the $e^{-}-e^{+}$fluid, and it increases and then decreases as $\xi$ increases. The behavior can be understood as follows. At the same $a$, as $\xi$ increases, the thermal energy increases, but at the same time, the rest-mass energy increases as well. As noted in Section 1, it is not the thermal energy, but the competition between the thermal energy and the rest-mass energy that makes a fluid relativistic. Consequently, for most values of $a, N$ increases for $\xi \lesssim 0.2$ and then decreases for $\xi \gtrsim 0.2$. For very low $a, N$ increases up to $\xi \sim 0.5$, and for very high $a, N$ increases up to $\xi \lesssim 0.1$. In summary, at a given temperature, the $e^{-}-e^{+}$fluid is most relativistic, but at a given sound speed, the $e^{-}-e^{+}$fluid is least relativistic and fluids with finite proton proportions are more relativistic.

\subsection{Equations of Motion}

The energy-momentum conservation equation (the first of Equation (3)) can be reduced to the relativistic Euler equation and the entropy equation. Under the steady state and radial flow assumptions, the equations of motion are given by

$$
u^{r} \frac{d u^{r}}{d r}+\frac{1}{r^{2}}=-\left(1-\frac{2}{r}+u^{r} u^{r}\right) \frac{1}{e+p} \frac{d p}{d r},
$$

and

$$
\frac{d e}{d r}-\frac{e+p}{n} \frac{d n}{d r}=0,
$$

along with the continuity equation (the second of Equation (3))

$$
\frac{1}{n} \frac{d n}{d r}=-\frac{2}{r}-\frac{1}{u^{r}} \frac{d u^{r}}{d r} .
$$

Here, we use the system of units where $G=M_{B}=c=1$, so that the units of length and time are $r_{g}=G M_{B} / c^{2}$ and $t_{g}=G M_{B} / c^{3}$. It is to be noted that in this system of units, the Schwarzschild radius or the radius of the event horizon is $r_{s}=2$. After some lengthy calculations, Equations (6a)-(6c) are then simplified to

$$
\frac{d v}{d r}=\frac{\left(1-v^{2}\right)\left[a^{2}(2 r-3)-1\right]}{r(r-2)\left(v-a^{2} / v\right)}
$$

and

$$
\frac{d \Theta}{d r}=-\frac{\Theta}{N}\left[\frac{2 r-3}{r(r-2)}+\frac{1}{v\left(1-v^{2}\right)} \frac{d v}{d r}\right],
$$

where the radial three-velocity is defined as $v^{2}=-u_{r} u^{r} /\left(u_{t} u^{t}\right)$.

For flows continuous along streamlines, Equations (7a) and (7b) admit the so-called regularity condition, or the critical point condition, or the sonic-point condition (Chakrabarti 1990) that is given by

$$
a_{c}=v_{c}
$$

and

$$
a_{c}^{2}=\frac{1}{2 r_{c}-3} \text {. }
$$

Here, $r_{c}$ is the sonic-point location. Hereafter, the quantities with subscript $c$ denote those at $r_{c}$. From Equation (5k), we 
know $a_{\max }=1 / \sqrt{3}$ (also see Figure 1(b)). Therefore, from Equation (8b), we have $r_{c} \geqslant 3$ (Blumenthal \& Mathews 1976). Since $d v / d r=\mathcal{N} / \mathcal{D} \rightarrow 0 / 0$ at $r_{c},(d v / d r)_{r_{c}}$ is obtained by the l'Hospital rule

$$
\left(\frac{d v}{d r}\right)_{r_{c}}=\frac{(d \mathcal{N} / d r)_{r_{c}}}{(d \mathcal{D} / d r)_{r_{c}}}
$$

where $\mathcal{N}$ and $\mathcal{D}$ are the numerator and denominator of Equation (7a). The above equation simplifies to

$$
\mathcal{A}\left(\frac{d v}{d r}\right)_{r_{c}}^{2}+\mathcal{B}\left(\frac{d v}{d r}\right)_{r_{c}}+\mathcal{C}=0
$$

where

$$
\begin{gathered}
\mathcal{A}=\left(2+\frac{1-N_{c} a_{c}^{2}+\left(\Theta_{c} / \Gamma_{c}\right)(d \Gamma / d \Theta)_{c}}{N_{c}\left(1-a_{c}^{2}\right)}\right) r_{c}\left(r_{c}-2\right), \\
\mathcal{B}=2 \frac{1-N_{c} a_{c}^{2}+\left(\Theta_{c} / \Gamma_{c}\right)(d \Gamma / d \Theta)_{c}}{N_{c} a_{c}},
\end{gathered}
$$

and

$$
\mathcal{C}=2 \frac{1-N_{c} a_{c}^{2}+\left(\Theta_{c} / \Gamma_{c}\right)(d \Gamma / d \Theta)_{c}}{N_{c} r_{c}}-2 a_{c}^{2}\left(1-a_{c}^{2}\right) .
$$

Equation (8d) has two roots. For radial flows, the roots are of the saddle type, where $(d v / d r)_{c}$ is real and $(d M / d r)_{c}$ is of opposite signs for the two roots. Here, $M=v / a$ is the Mach number. Moreover, the two roots can be either of the acceleration type (Atype), where $(d v / d r)_{c}$ is of opposite signs, or of the deceleration type (D-type), where $(d v / d r)_{c}$ is negative for both roots. In the A-type, both the acceleration and wind flows accelerate at the sonic point. On the other hand, in the D-type, only the accretion flows accelerate, while the wind flows decelerate at the sonic point.

By substituting the quantities at the sonic point, Equation ( $7 b$ ) gives the temperature gradient at the sonic point

$$
\left(\frac{d \Theta}{d r}\right)_{r_{c}}=-\frac{\Theta_{c}}{N_{c}}\left[\frac{2 r_{c}-3}{r_{c}\left(r_{c}-2\right)}+\frac{1}{v_{c}\left(1-v_{c}^{2}\right)}\left(\frac{d v}{d r}\right)_{r_{c}}\right] .
$$

Finally, by integrating the equations of motion, we get the relativistic Bernoulli equation (Lightman et al. 1975)

$$
\mathcal{E}=\frac{(f+2 \Theta) u_{t}}{(2-\xi+\xi / \eta)}
$$

where $\mathcal{E}$ is the Bernoulli parameter or is also known as the specific energy of flows. Since we assume adiabatic flows without heating and cooling, $\mathcal{E}$ is a constant of motion.

\subsection{Procedure to Get Global Solutions}

Combining Equations (8b) and (5k) gives $\Theta_{c}$ in terms of $r_{c}$ and $\xi$. Combining it with Equation (9) gives a formula involving $r_{c}, \mathcal{E}$, and $\xi$ (Chakrabarti 1990, 1996b; Fukumura \& Kazanas 2007). If $\mathcal{E}$ and $\xi$ are given, then $r_{c}$ is computed from the formula. Once $r_{c}$ is known, all the quantities at $r_{c}$, e.g., $\Theta_{c}$, $v_{c},(d v / d r)_{r_{c}},(d \Theta / d r)_{r_{c}}$, etc., are computed from Equations (8a)-(8h). Then Equations (7a) and (7b) are integrated, starting from $r_{c}$, once inward and then outward, to obtain the global, transonic solutions of spherical flows around black holes. By this way, we can obtain two parameter $(\mathcal{E}, \xi)$ family of accretion and wind solutions.

\section{SONIC-POINT PROPERTIES}

In the transonic flows we study, the sonic point plays an important role. So before we present global solutions in the next section, we first investigate the properties of the sonic point in this section. Understanding the sonic-point properties will allow us to have an idea of the nature of global flow structures.

The sonic point location, $r_{c}$, that is computed as a function of $\mathcal{E}$ and $\xi$, is presented in Figure 2(a). Corresponding to each set of $\mathcal{E}$ and $\xi$ values, there exists a unique $r_{c}$. Each curve, which is given as a function of $\mathcal{E}$, is for a different value of $\xi$. If a flow is more energetic with larger $\mathcal{E}$, it is characterized by a smaller value of $r_{c}$. However, at the same $\mathcal{E}, r_{c}$ is smallest for the $e^{-}-e^{+}$fluid (solid line). The value of $r_{c}$ increases for $\xi \lesssim 0.2$, and then starts to decrease for larger $\xi$. In other words, if fluids of the same $\mathcal{E}$ but different $\xi$ are launched at a large distance away from a black hole, then the $e^{-}-e^{+}$fluid crosses the sonic point closest to the event horizon, compared to the fluids of finite proton proportion. Alternatively, at the same $r_{c}$, $\mathcal{E}$ is smallest for the $e^{-}-e^{+}$fluid, and it increases up to $\xi \sim 0.2$ and then decreases for $0.2 \lesssim \xi \leqslant 1$. Although for the same $r_{c}$ the $e^{-}-p^{+}$(dotted line) is not most energetic, it is definitely more energetic than the $e^{-}-e^{+}$fluid. Since fluids of different composition are energetically quite different at the same $r_{c}$, or conversely fluids of different composition but the same $\mathcal{E}$ form the sonic point at widely different $r_{c}$, it is expected that the global solutions of accretion and wind flows would be quantitatively and qualitatively different, depending upon the composition of fluids.

In Figures 2(b) and (c), we show $T_{c}$ and $N_{c}$ as a function of $r_{c}$. Equation (8b) tells that the sound speed at the sonic point, $a_{c}$, is fixed, once $r_{c}$ is determined ( $a_{c}$ implicitly depends on $\mathcal{E}$ and $\xi$ through $r_{c}$ ). So plotting any variable as a function of $r_{c}$ is equivalent to plotting it as a function of $a_{c}$. As noted above, at the same $a_{c}$, fluids composed of lighter particles are colder. Therefore, in Figure 2(b), at the same $r_{c}$, the temperature is lowest for the $e^{-}-e^{+}$fluid, progressively gets higher for fluids with larger proton proportions, and the maximum temperature is for the $e^{-}-p^{+}$fluid. However, as noted before, higher $T_{c}$ does not necessarily ensure higher $N_{c}$ (i.e., more relativistic fluids). In Figure 2(c), at the same $r_{c}$, the $e^{-}-e^{+}$fluid has the lowest $N_{c}$, that is, it is least relativistic. In the range of a few $\lesssim r_{c} \lesssim 100$, at the same $r_{c}, N_{c}$ increases as the proton proportion increases for $\xi \lesssim 0.2$, and then starts to decrease for $0.2 \lesssim \xi \leqslant 1$. This is a consequence of the competition between the thermal energy and the rest-mass energy, as discussed in connection with Figures 1(c). In order to make the point even clearer, in Figure 2(d), we show $N_{c}$ as a function of $\xi$ for a wide range of values of $r_{c}$. Each curve with a single value of $r_{c}$ signifies fluids of different composition but the same sound speed at the same sonic point. $N_{c}$ tends to peak at some values of $\xi$, where the thermal contribution with respect to the rest-mass energy contribution peaks. For small values of $r_{c}$ (i.e., large $a_{c}$ 's), a small increase of $\xi$ causes the thermal contribution to peak. For large values of $r_{c}$ (i.e., small $a_{c}$ 's), large proton proportions are needed to achieve the same.

As discussed in Section 2.3, the roots of Equation (8d) are either of the A-type or of the D-type. At small values of $r_{c}$, the nature of the sonic point is of the A-type. It is because if the sonic point form closer to the central object, the flow is hotter at the sonic point (Figure 2(b)), and in the wind that is thermally driven, the flow tends to accelerate at the sonic point. But beyond a limiting value, say $r_{c \ell}$, the nature changes from 

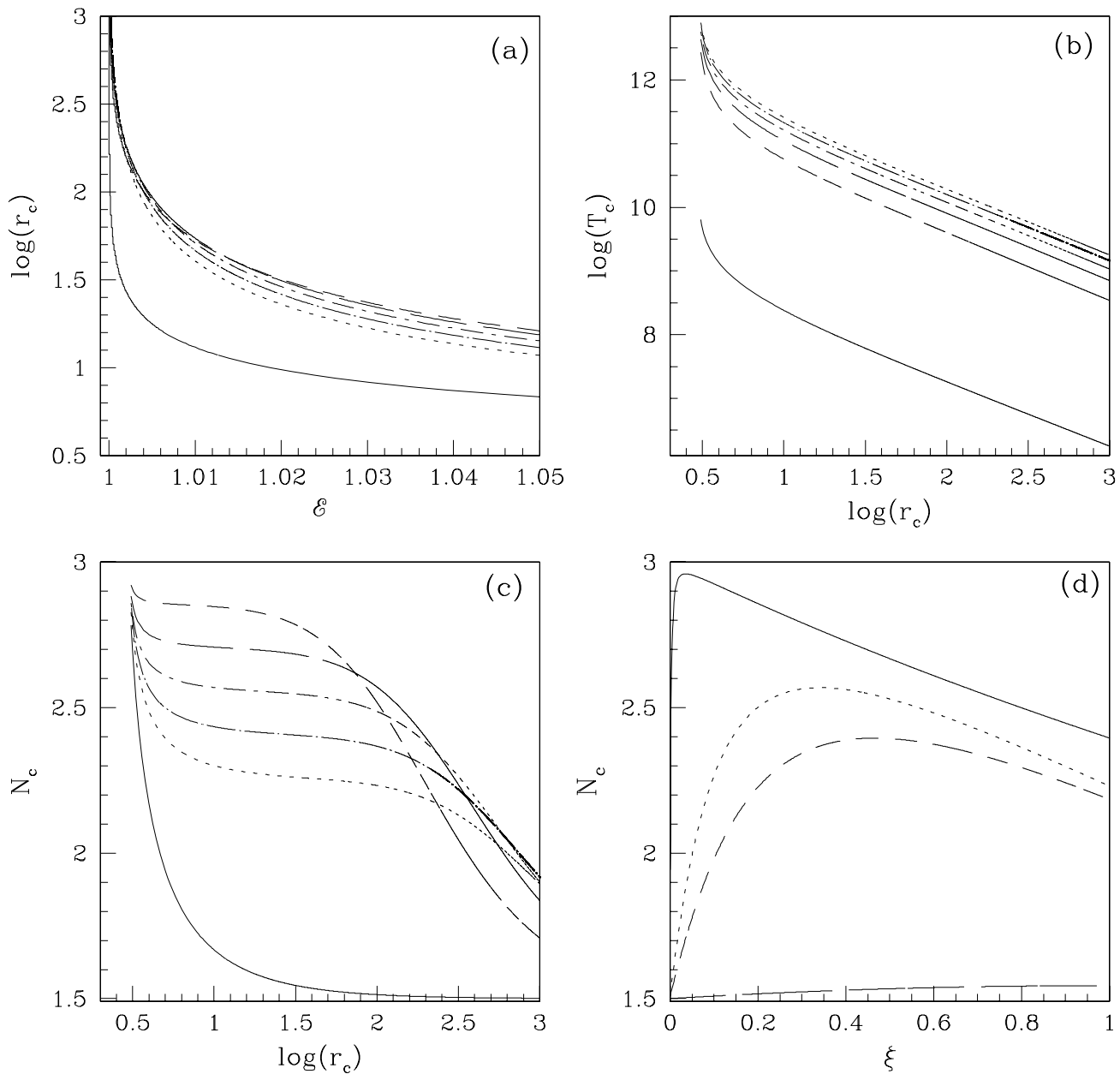

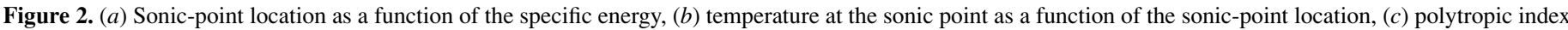

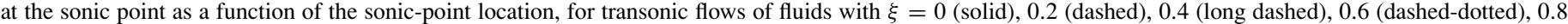

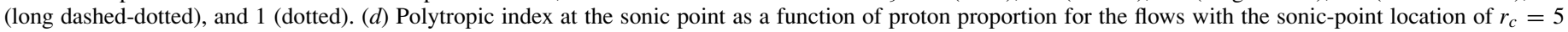
(solid), 105 (dotted), 205 (dashed), and 13, 505 (long dashed).
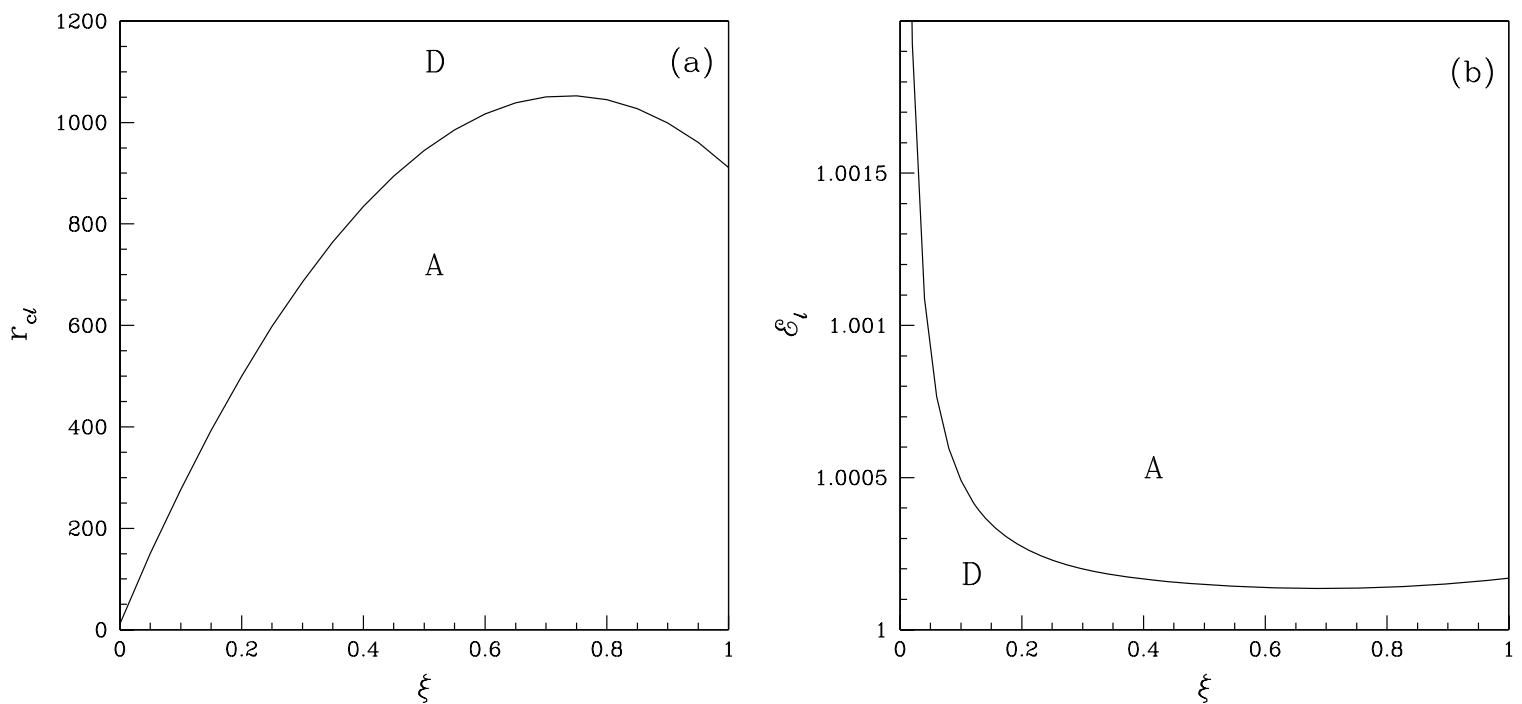

Figure 3. Limiting values of $(a)$ the sonic-point location and $(b)$ the specific energy, which divide the domain of the A-type sonic-point roots from that of the D-type sonic-point roots, as a function of proton proportion.

the A-type to the D-type, where the wind flow decelerates at the sonic point. In Figure 3(a), $r_{c \ell}$ is plotted as a function of $\xi$. Since at a given $r_{c}$ the $e^{-}-e^{+}$fluid is thermally least relativistic, $r_{c \ell}$ is smallest for the fluid. The limit $r_{c \ell}$ increases with $\xi$. However, since increasing $\xi$ makes fluids "heavy" too, $r_{c \ell}$ peaks around $\xi \sim 0.75$. In Figure 3(b), we plot the limiting values of $\mathcal{E}$ 

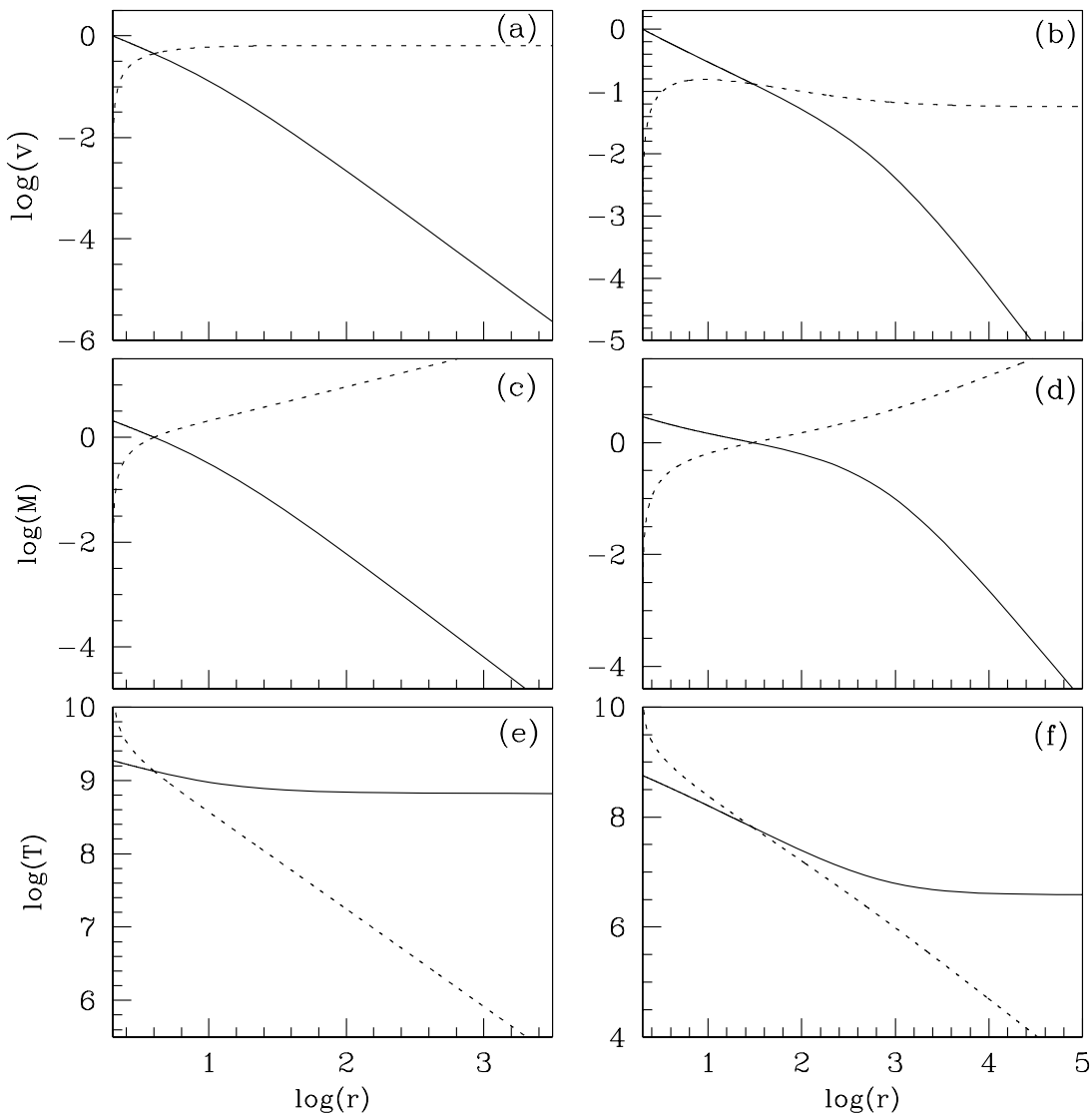

Figure 4. Examples of transonic accretion (solid) and wind (dotted) solutions of the A-type with the sonic point at $r_{c}=4\left(\mathcal{E}=1.3, N_{c}=2.163\right)$ (left panels) and of the D-type with the sonic point at $r_{c}=30\left(\mathcal{E}=1.0016, N_{c}=1.548\right)$ (right panels). The $e^{-}-e^{+}$fluid $(\xi=0)$ is considered. The radial three-velocity (top), Mach number (middle), and temperature (bottom) are shown as a function of radius.

corresponding to $r_{c \ell}, \mathcal{E}_{\ell}$, as a function of $\xi$, such that for $\mathcal{E}>\mathcal{E}_{\ell}$ the nature of the sonic point is of the A-type, for $\mathcal{E}<\mathcal{E}_{\ell}$ it is of the D-type.

\section{SPHERICAL ACCRETION AND WIND SOLUTIONS}

In this section, we present the global solutions of Equations (7a) and (7b) that were obtained with the procedure described in Section 2.4. In Figure 4, we first compare typical accretion and wind solutions of the $\mathrm{A}$ and D-types for the $e^{-}-e^{+}$fluid. The solutions of the A-type in the left panels have the sonic point at $r_{c}=4$, inside $r_{c \ell}$, while those of the D-type in the right panels have $r_{c}=30$, beyond $r_{c \ell}$ (Figure 3(a)). The accretion solutions (solid curves) are characterized by supersonic flows at the inner boundary and subsonic flows at the outer boundary (Figures 4(c) and (d)). The wind solutions (dotted curves), on the other hand, have subsonic flows at the inner boundary and supersonic flows at the outer boundary. The accretion flows around black holes necessarily accelerate inward. However, the wind flows may accelerate (Figure 4(a)) or decelerate (Figure 4(b)) outward. The wind solutions considered in this paper are thermally driven. These winds are very hot at the base, and are powered by the conversion of the thermal energy into the kinetic energy. It can be shown from Equation (7b) that

$$
-\frac{d \Theta}{d r} \leqslant \frac{\Theta}{N}\left[\frac{2 r-3}{r(r-2)}\right] \Rightarrow \frac{d v}{d r} \leqslant 0 .
$$

In other words, if the outward thermal gradient is weaker than the gravity, the outflow can decelerate. For the wind with $r_{c}=30$
(Figures 4(b), (d), and (f)), $-d \Theta / d r \sim(\Theta / N)(2 r-3) /[r(r-2)]$ at $r \sim 9.16$, exactly where the outflow starts to decelerate. However, the wind velocity will reach an asymptotic value at $r \rightarrow$ large, since $-d \Theta / d r \sim(\Theta / N)(2 r-3) /[r(r-2)] \sim 0$ at large distances from the black hole. Similar relation between the gradients at the sonic point will determine the nature of the sonic point. It may be noted that at $r_{c} \geqslant r_{c \ell}$ (Figures 3(a) and (b)), such relation between $(d v / d r)_{c}$ and $(d \Theta / d r)_{c}$ is satisfied. Regardless of accretion/wind or the type, the temperature decreases with increasing $r$ (Figures 4(e) and (f)).

We note that the winds in our solutions are too weak to be the precursor of astrophysical jets, until and unless other accelerating processes like those caused by magnetic fields or disk radiation are considered (Chattopadhyay 2005). In fact, we checked that it is not possible to generate the terminal speed much greater than $\sim 0.8 c$ for purely thermally driven winds, such as the ones that are considered in this paper. It is also to be noted that our D-type, wind solution is not an example of "breeze." A breeze is always subsonic, while the wind here is transonic, albeit decelerating.

In the previous figure, we have compared the solutions with the same $\xi(=0)$ but different $r_{c}$. In Figure 5, we compare the solutions with the same $r_{c}(=20)$ but different $\xi$. As shown in Figure 2(a), even for the same $r_{c}$, the specific energy is different for fluids of different $\xi$. Furthermore, the polytropic index at the sonic point is different too $\left(N_{c}=1.547\right.$ in Figure 5(a), $N_{c}=2.626$ in Figure 5(b), and $N_{c}=2.271$ in Figure 5(c)). Therefore, even if we fix the sonic point (and therefore $a_{c}$ ), the flow structure and energetics are different for fluids with 

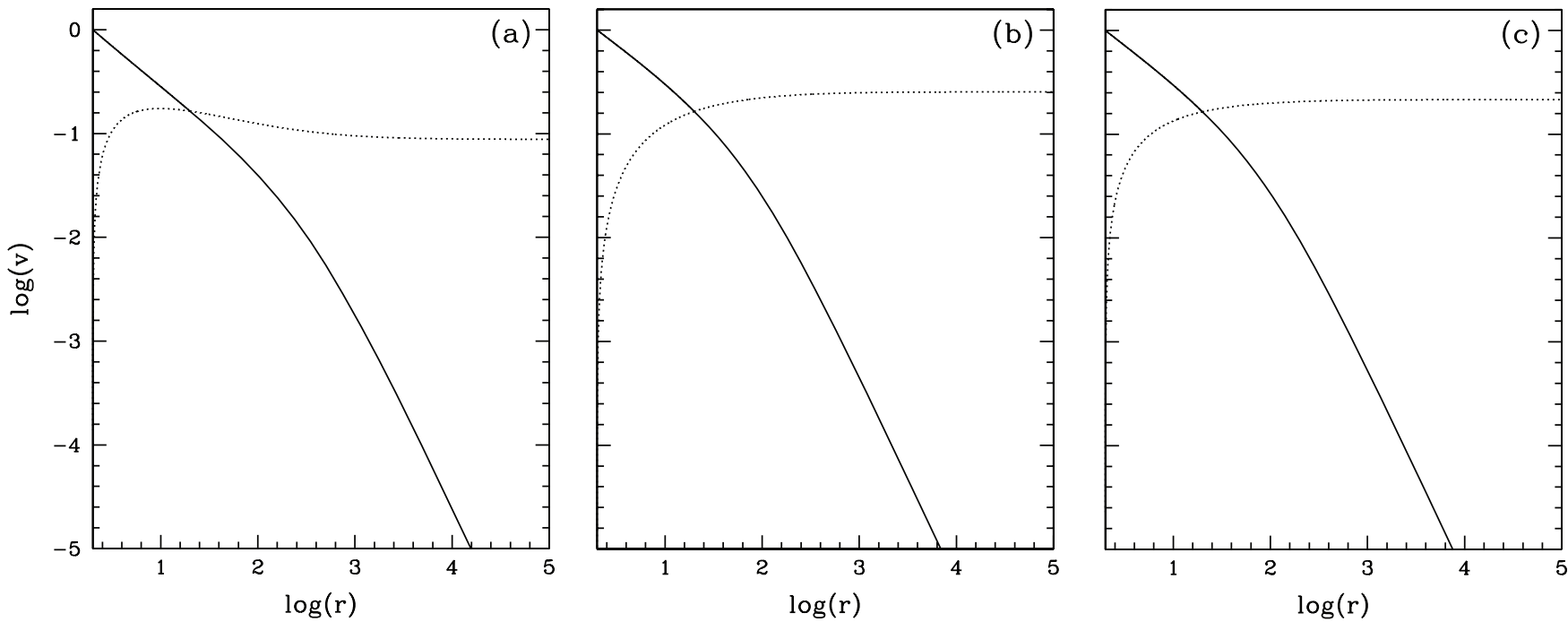

Figure 5. Comparison of accretion (solid) and wind (dotted) solutions with the same sonic-point location of $r_{c}=20$ but different proton proportions of (a) $\xi=0$ $(\mathcal{E}=1.0039)$, (b) $\xi=0.5(\mathcal{E}=1.0337)$, and (c) $\xi=1(\mathcal{E}=1.0239)$. The radial three-velocity is shown as a function of radius.

different $\xi$. In these particular solutions, the $e^{-}-e^{+}$fluid is not hot enough to drive an accelerating wind (Figure 5(a)), while the fluids with significant protons can do so. As in the previous case of decelerating wind solution (i.e., Figure 4(b)), in the present case the $e^{-}-e^{+}$fluid first accelerates and then starts to decelerate at $r \sim 9.86$. The velocity profile eventually tapers off to an asymptotic value at large distances away from the black hole. It has been shown in Figure 2(b) that at the same $r_{c}$, adding protons increases the temperature at the sonic point. Larger temperature gradient causes winds of finite proton proportion to be accelerated at the sonic point (Figure 5(b)). It is seen that beyond a critical value, the increase in $\xi$ increases the inertia which reduces the wind speed, as is vindicated by Figures 5(b) and (c). It is to be remembered that, the D-type sonic point is a reality for fluids of any $\xi$, provided $r_{c} \gtrsim r_{c \ell}$.

Although the wind solutions are noticeably different depending on $\xi$, there seems to be only small difference in the velocity profile of the accretion solutions. Henceforth, we concentrate only on accretion solutions. Such small difference in $v$ in accretion solutions is expected. The accretion is generated mostly by the inward pull of the gravity, which gives the unique inner boundary condition for black holes, i.e., $v=1$ at $r=2$, regardless of other considerations. The pressure gradient changes the profile of $v$ too. Since the composition of fluids determines the thermal state, it influences the profile of $v$, but the effect is not the dominant one.

In Figure 6, we compare the accretion solutions with the same $\mathcal{E}(=1.015)$ but different $\xi$. As noted below Equation $(9), \mathcal{E}$ is a constant of motion. For $r \rightarrow \infty$, as $u_{t} \rightarrow 1$, we have $\mathcal{E} \rightarrow h_{\infty}$, where

$$
h_{\infty}=\left[\frac{e+p}{\rho}\right]_{\infty}=\left[\frac{f+2 \Theta}{2-\xi+\xi / \eta}\right]_{\infty}
$$

is the specific enthalpy at infinity. Equation (11) tells us that at large distances from black holes, for the same $\mathcal{E}, T$ is large if $\xi$ is large. Hence, fluids with larger $\xi$ are hotter to start with. Therefore, even for fluids with the same $\mathcal{E}$, the solutions are different if $\xi$ is different. Figure 6(a) shows the velocity profile as a function of $r$. Here, the difference in $v$ for fluids with different $\xi$ is evident, albeit not big as pointed above. Figures 6(b)-(d) show the mass density, temperature, and polytropic index. To compute the mass density, we need to supply the mass accretion rate, which is given as

$$
\dot{M}=4 \pi r^{2} u^{r} \rho
$$

from Equation (6c). The mass density in Figure 6(b) was computed for $M_{B}=10 M_{\odot}$ and $\dot{M}=0.1 \dot{M}_{\text {Edd }}$, where $\dot{M}_{\text {Edd }}$ is the Eddington rate of accretion. The difference in $T$ and $N$ for fluids with different $\xi$ is more pronounced. The $e^{-}-e^{+}$fluid is slowest, densest (for the same $\dot{M}$ ), coldest, and least relativistic. The $e^{-}-p^{+}$fluid is more relativistic than the $e^{-}-e^{+}$fluid. But the most relativistic fluid is the one with the intermediate value of $\xi$. It is interesting to note that except for the $e^{-}-e^{+}$fluid, $N$ is a slowly varying function of $r$ for the other two fluids. Does this mean it would be sufficient to adopt the fixed $\Gamma$ EoS with appropriate values of $\Gamma$ ?

Finally in Figure 7, we compare the accretion solutions with the same temperature at large distances but different $\xi$. All the fluids start with $T=T_{\text {out }}=1.3 \times 10^{9} \mathrm{~K}$ at $r=r_{\text {out }}=2000$. Again the mass density was computed for $M_{B}=10 M_{\odot}$ and $\dot{M}=0.1 \dot{M}_{\text {Edd }}$. It is to be noted that the fluids starting with the same $T_{\text {out }}$ but different $\xi$ have different specific energies. Hence, the velocity at the outer boundary is different too. As shown in Figure 7(a), in these particular solutions, the $e^{-}-e^{+}$fluid starts with a velocity substantially different from those of the other two fluids, so the resulting velocity profile is substantially different. From Figure 7(d), it is clear that there are significant variations in $N$ for all the fluids. The $e^{-}-e^{+}$fluid starts with the largest $N$. It is because at the same temperature, the $e^{-}-e^{+}$fluid is thermally most relativistic. The behavior of $N$ can be traced back to Figure 1(a). For instance, the variations in $N$ tend to flatten at $T \gtrsim 10^{10} \mathrm{~K}$. In Figure 7(c), for the fluids with $\xi=0.5$ and $1, T \lesssim 10^{10} \mathrm{~K}$ for $r \gtrsim 100$ and $T \gtrsim 10^{10} \mathrm{~K}$ for $r \lesssim 100$. So significant variations are expected in $N$ at $r \gtrsim 100$, while the variations flatten at $r<100$. Similar considerations will explain the variations in $N$ for the $e^{-}-e^{+}$fluid. From Figure $7(d)$, it is clear that we need to adopt a relativistically correct EoS (Equation (4c) or (4d)), instead of the EoS with a fixed $\Gamma$, in order to capture the proper thermal properties of flows around black holes.

In this section, we have shown that fluids with different composition can result in dramatically different accretion and 

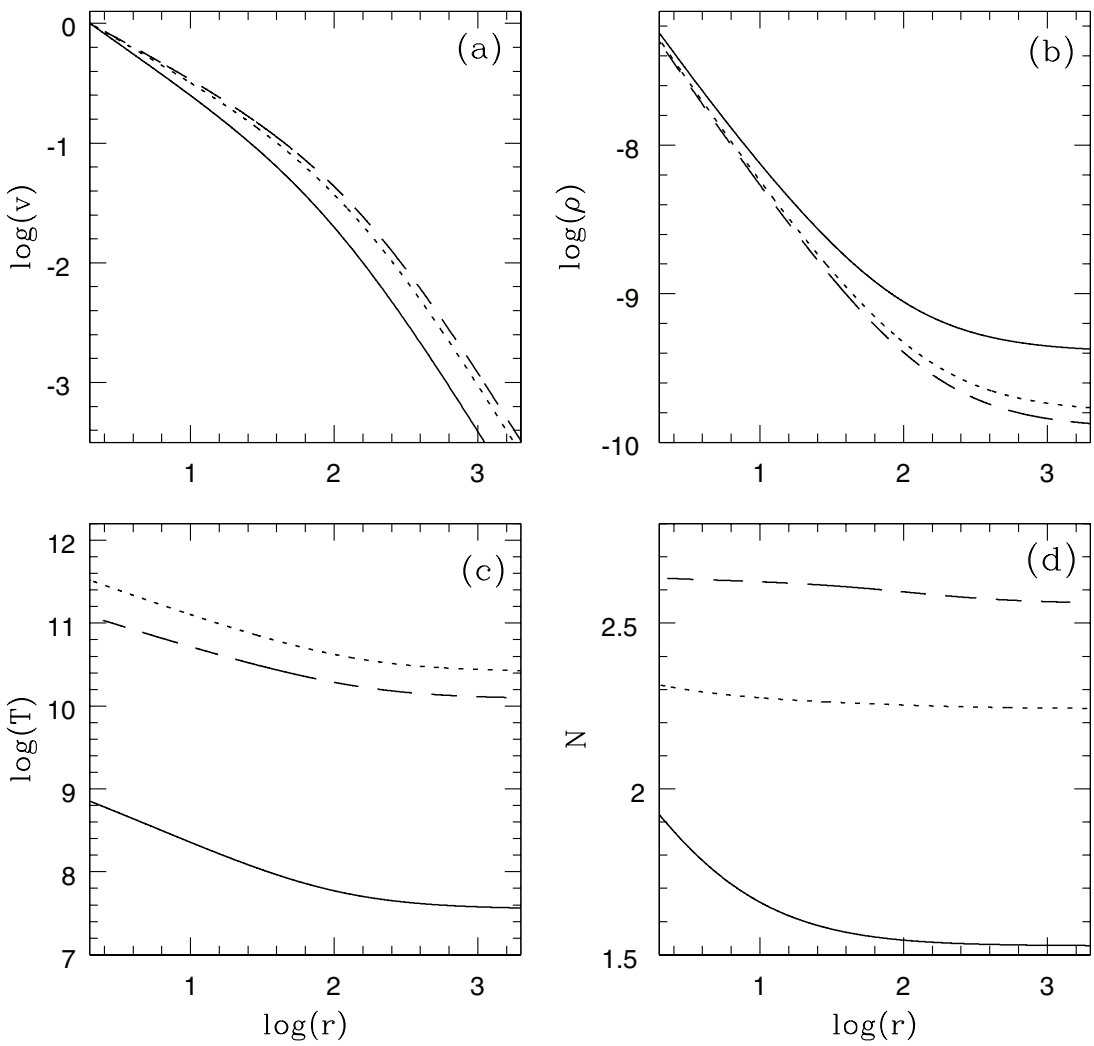

Figure 6. Comparison of accretion solutions with the same specific energy of $\mathcal{E}=1.015$ but different proton proportions of $\xi=0$ (solid), $\xi=0.5$ (dashed), and $\xi=1$ (dotted). The sonic-point locations are $r_{c}=11.0$ for $\xi=0, r_{c}=38.267$ for $\xi=0.5$, and $r_{c}=28.972$ for $\xi=1$. The radial three-velocity $(a)$, mass density (b), temperature $(c)$, and polytropic index $(d)$ are shown as a function of radius. The mass density was computed assuming the black hole of $M_{B}=10 M_{\odot}$ and the accretion rate of $\dot{M}=0.1 \dot{M}_{\mathrm{Edd}}$, and is given in units of $\mathrm{g} \mathrm{cm}^{-3}$.
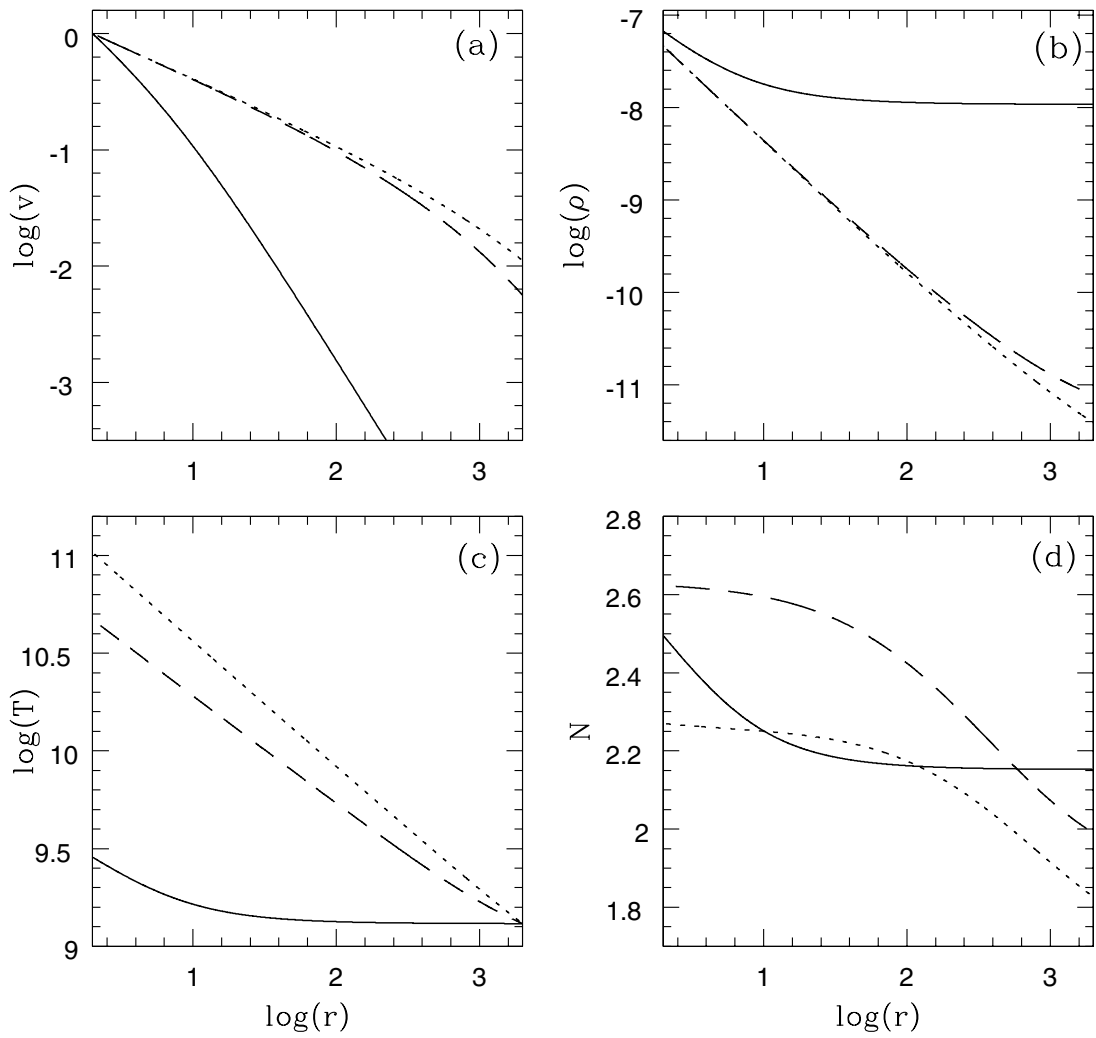

Figure 7. Comparison of accretion solutions with the same temperature of $T_{\text {out }}=1.3 \times 10^{9} \mathrm{~K}$ at the outer boundary $r_{\text {out }}=2000$ but different proton proportions of $\xi=0$ (solid), $\xi=0.5$ (dashed), and $\xi=1$ (dotted). The sonic-point location and the specific energy are $r_{c}=3.5$ and $\mathcal{E}=1.6322$ for $\xi=0, r_{c}=333.3$ and $\mathcal{E}=1.0008$ for $\xi=0.5$, and $r_{c}=806.4$ and $\mathcal{E}=1.0002$ for $\xi=1$. The radial three-velocity $(a)$, mass density $(b)$, temperature $(c)$, and polytropic index $(d)$ are shown as a function of radius. The mass density was computed assuming the black hole of $M_{B}=10 M_{\odot}$ and the accretion rate of $\dot{M}=0.1 \dot{M}$ Edd , and is given in units of $\mathrm{g} \mathrm{cm}^{-3}$. 

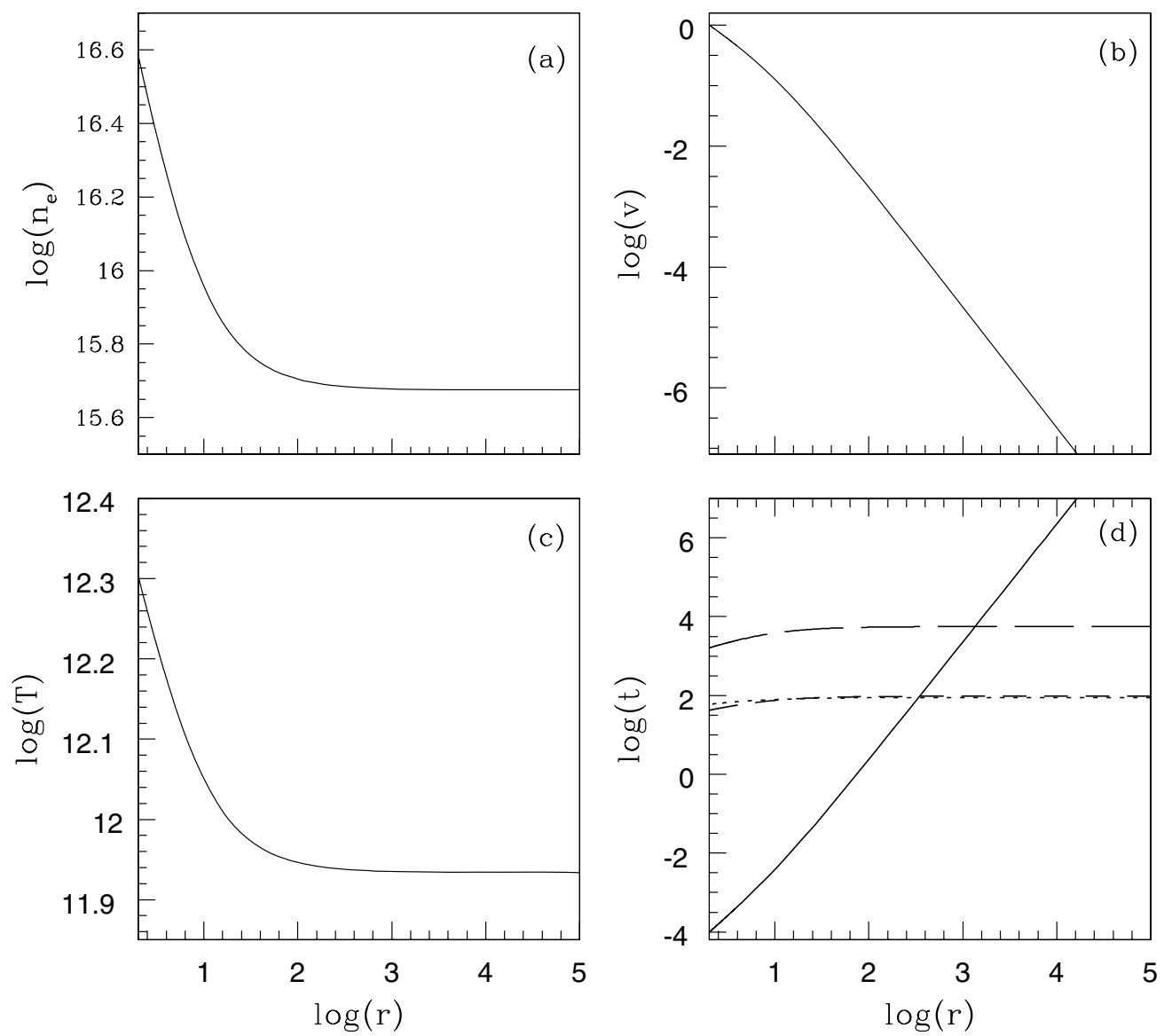

Figure 8. (a) Electron number density, $(b)$ radial three-velocity, and (c) temperature as a function of radius in an accreting flow of the $e^{-}-p^{+}$fluid $(\xi=1)$ with $\mathcal{E}=1.5247 . \dot{M}=0.1 \dot{M}_{\text {Edd }}$ onto a black hole of $M_{B}=10 M_{\odot}$ was assumed. (d) Comparison of various timescales of the same flow. Different curves represent the accretion time $t_{\mathrm{dyn}}$ (solid), the electron-electron relaxation time $t_{\mathrm{ee}}$ (dotted), the proton-proton relaxation time $t_{\mathrm{pp}}$ (dashed), and the electron-proton relaxation time $t_{\text {ep }}$ (long-dashed).

wind flows, even if they have the same sonic point or the same specific energy, or they start with the same temperature at large distances from black holes. So not just adopting a correct EoS, but incorporating the effects of fluid composition into the EoS (see Equation (5e)) should be also important in describing such flows.

\section{VALIDITY OF EOS}

In Section 2, we have made the following assumptions for our EoS (Equation (5e)); fluids are in equilibrium, i.e., (1) the distribution of the constituent particles is relativistically Maxwellian and (2) the multicomponents are of single temperature. However, it is not clear whether the conditions are satisfied. Most astrophysical fluids, unlike the terrestrial ones, consist of charged particles, which are collisionless, and so held together by magnetic fields. The constituent particles, on the other hand, exchange energies, and become relaxed mostly through the Coulomb interaction, which is a slow process in collisionless plasmas. In addition, most of the heating processes, such as viscosity and shock heating, are likely to affect protons. However, it is mainly the electrons which radiate. So the energy exchange between electrons and protons should operate, and eventually govern the thermal properties of fluids.

Let $t_{\mathrm{ee}}$ be the electron-electron relaxation timescale, $t_{\mathrm{pp}}$ be the proton-proton relaxation timescale, and $t_{\mathrm{ep}}$ be the electronproton relaxation timescale. And let $t_{\text {prob }}$ be the timescale of problem, such as the dynamical timescale, or the heating and/ or cooling timescale. Only if $t_{\mathrm{ee}}<t_{\mathrm{prob}}$ and $t_{\mathrm{pp}}<t_{\mathrm{prob}}$, electrons and protons will separately attain the Maxwellian distributions. And only if $t_{\mathrm{ep}}<t_{\text {prob }}$, electrons and protons will relax to single temperature.

To verify the assumptions for our EoS, in this section, we compare the relaxation timescales with the dynamical or accretion timescale $\left(t_{\mathrm{dyn}}=r / v\right)$ for an accretion solution. We consider the temperature range where protons are thermally nonrelativistic while electrons are relativistic. In most of our solutions in the previous section, the computed temperature favors this range. The relativistic electron-electron interaction timescale was derived by Stepney (1983),

$$
t_{\mathrm{ee}}=\frac{8 k^{2}}{\left(m_{e} c^{2}\right)^{2} \sigma_{T} c \ln \Lambda} \frac{T^{2}}{n_{e^{-}}} .
$$

The timescale for the nonrelativistic proton-proton interaction is given in Spitzer (1962),

$$
t_{\mathrm{pp}}=\frac{4 \sqrt{\pi} k^{3 / 2}}{\ln \Lambda\left(m_{p} c^{2}\right)^{3 / 2} \sigma_{T} c}\left(\frac{m_{p}}{m_{e}}\right)^{2} \frac{T^{3 / 2}}{n_{p^{+}}} .
$$

The relativistic electron-proton interaction timescale was also derived by Stepney (1983),

$$
t_{\mathrm{ep}}=2\left(\frac{m_{p}}{m_{e}}\right)\left(\frac{\kappa}{m_{e} c^{2}}\right) \frac{1}{\sigma_{T} c} \frac{T}{n_{p^{+}}} .
$$

We present the electron number density, $n_{e^{-}}$(Figure 8(a)), the three-velocity $v$ (Figure 8(b)) and the temperature $T$ (Figure 8(c)) 
of the accretion solution for the $e^{-}-p^{+}$fluid with $\mathcal{E}=1.5247$. The electron number density was computed for $M_{B}=10 M_{\odot}$ and $\dot{M}=0.1 \dot{M}_{\text {Edd. }}$. In Figure 8(d), various timescales are compared. All the relaxation timescales were calculated for the solution of single temperature. To our surprise, it is clear that the accretion flow in the figure is "too fast," such that various relaxation timescales are longer than the accretion timescale at least within few tens of $r_{s}$. The implication of it is not clear, however. For instance, in relativistic plasmas, the constituent particles can be relaxed through the interactions with magnetic fields, too. But the relaxation will depend on the details of field configuration, such as the strength and the topology. Since we ignore in this study magnetic fields as well as other processes such as nonconservative ones, we leave this issue of the validity of our EoS for future studies.

\section{DISCUSSION AND CONCLUDING REMARKS}

In this paper, we have investigated the effects of fluid composition on the solutions of accretion and wind flows onto black holes. In order to elucidate the effects, we have considered a very simple model of spherical flows onto Schwarzschild black holes, and nonconservative processes and magnetic fields have been ignored.

First, we have suggested an approximate EoS for multicomponent fluids in Equation (5e), and studied the thermal properties of fluids with the EoS. Three temperature ranges have been categorized; for $k T<m_{e} c^{2}$, any type of fluids are thermally nonrelativistic, for $k T>m_{p} c^{2}$, any type of fluids are thermally relativistic, and for $m_{e} c^{2}<k T<m_{p} c^{2}$, the degree to which fluids are relativistic is determined by the composition of the fluids as well as the temperature (Figure 1(a)). Then we have shown that although at the same temperature the $e^{-}-e^{+}$fluid is most relativistic (Figure 1(a)), at the same sound speed it is least relativistic (Figure 1(c)), compared to the fluids with protons. It is because whether a fluid is relativistic or not depends on the competition between the thermal energy and the rest-mass energy of the fluid.

The thermal properties of fluids carry to the sonic-point properties. The sound speed at the sonic point, $a_{c}$, explicitly depends only on the sonic-point location, $r_{c}$ (it implicitly depends on the specific energy, $\mathcal{E}$, and the proton proportion, $\xi$, through $r_{c}$ ). Therefore, comparing the thermodynamic quantities at the same $r_{c}$ is equivalent to comparing those quantities at the same $a_{c}$. We have shown that at the same $r_{c}$, the $e^{-}-e^{+}$fluid is least relativistic, and a fluid with a finite $\xi$ is most relativistic (Figures 2(c) and (d)).

Then, we have presented the global solutions of accretion and wind flows for the same $r_{c}$ but different $\xi$, for the same $\mathcal{E}$ but different $\xi$, and for the same $T$ at large distances from black holes but different $\xi$. In all the cases, the flows can be dramatically different, if the composition is different. This asserts that the effects of fluid composition are important in the solutions, and hence, incorporating them properly into the solutions through the EoS is important.

Lastly, we have noted that the EoS in Equation (5e) is based on the assumptions that the distribution of the constituent particles is relativistically Maxwellian and the multicomponents are of single temperature. However, at the same time, we have pointed out that while the Coulomb relaxation times are normally shorter than the dynamical time far away from black holes, close to black holes they can be longer. It means that close to black holes, the assumptions for the EoS can be potentially invalidated. The implication of it needs to be understood, and we leave further consideration of this issue for future studies.

The work of D.R. was supported by the Korea Research Foundation grant funded by the Korean Government (MOEHRD) (KRF-2007-341-C00020).

\section{REFERENCES}

Barai, P., Das, T. K., \& Wiita, P. 2006, ApJ, 613, L49

Biretta, J. A., Sparks, W. B., \& Macchetto, F. 2003, ApJ, 520, 621

Blumenthal, G. R., \& Mathews, W. G. 1976, ApJ, 203, 714

Bondi, H. 1952, MNRAS, 112, 195

Chakrabarti, S. K. 1990, Theory of Transonic Astrophysical Flows (Singapore: World Scientific)

Chakrabarti, S. K. 1996a, MNRAS, 283, 325

Chakrabarti, S. K. 1996b, Phys. Rep., 266, 229

Chandrasekhar, S. 1938, An Introduction to the Study of Stellar Structure (NewYork: Dover)

Chattopadhyay, I. 2005, MNRAS, 356, 145

Corbel, S., Nowak, M. A., Fender, R. P., Tzioumis, A. K., \& Markoff, S. 2003, A\&A, 400, 1007

Das, T. K. 2001, A\&A, 376, 697

Das, T. K. 2002, MNRAS, 330, 563

Ferrari, A., \& Trussoni, E. 1985, ApJ, 294, 397

Fukumura, K., \& Kazanas, D. 2007, ApJ, 669, 85

Lightman, A. P., Press, W. H., \& Teukolsky, S. A. 1975, Problem Book in Relativity and Gravitation (Princeton, NJ: Princeton Univ. Press)

Mandal, I., Ray, A. K., \& Das, T. K. 2007, MNRAS, 378, 1400

Mathews, W. G. 1971, ApJ, 165, 147

Meliani, Z., Sauty, C., Tsinganos, K., \& Vlahakis, N. 2004, A\&A, 425, 773

Michel, F. C. 1972, Ap\&SS, 15, 153

Mignone, A., Plewa, T., \& Bodo, G. 2005, ApJS, 160, 199

Rózańska, A., \& Czerny, B. 2000, A\&A, 360, 1170

Ryu, D., Chattopadhyay, I., \& Choi, E. 2006, ApJS, 166, 410

Spitzer, L. 1962, Physics of Fully Ionized Gases (New York: Wiley)

Stepney, S. 1983, MNRAS, 202, 467

Synge, J. L. 1957, The Relativistic Gas (Amsterdam: North-Holland)

Taub, A. H. 1948, Phys. Rev., 74, 328

Woosley, S. E. 1993, ApJ, 405, 273 